Article

\title{
Application of Terahertz Spectroscopy to Rubber Products: Evaluation of Vulcanization and Silica Macro Dispersion
}

\author{
Yasuyuki Hirakawa ${ }^{1, *}$, Yuki Yasumoto ${ }^{1}$, Toyohiko Gondo ${ }^{1}$, Ryota Sone ${ }^{2}$, Toshiaki Morichika ${ }^{2}$, \\ Takakazu Minato ${ }^{2}$ and Masahiro Hojo ${ }^{2}$ \\ 1 National Institute of Technology (KOSEN), Kurume College, Kurume 830-8555, Japan; \\ yasugr4@gmail.com (Y.Y.); gonmota.9411@gmail.com (T.G.) \\ 2 Advanced Materials Division, Bridgestone Corporation, Kodaira 187-8531, Japan; \\ ryota.sone@bridgestone.com (R.S.); toshiaki.morichika1@bridgestone.com (T.M.); \\ takakazu.minato@bridgestone.com (T.M.); masahiro.hojo@bridgestone.com (M.H.) \\ * Correspondence: hirakawa@kurume-nct.ac.jp; Tel.: +81-942-35-9381
}

Received: 27 March 2020; Accepted: 18 April 2020; Published: 20 April 2020

\begin{abstract}
Industrial applications of terahertz $(\mathrm{THz})$ technology are becoming more widespread. In particular, novel evaluation methods for essential rubber products are being developed. THz absorbance spectra of various rubber polymers and reagents enable visualization of filler dispersions and vulcanization reactions. Here, improved visualization of the vulcanization reaction in thick rubber samples is discussed. Silica macro-dispersion is also analyzed because it is a general filler in automobile tires and has been difficult to evaluate with conventional techniques.
\end{abstract}

Keywords: THz-TDS; rubber; vulcanization; silica dispersion

\section{Introduction}

Terahertz (THz) waves are located in the electromagnetic band between the far-infrared and the microwave regions corresponding to the fingerprint region of some materials and can exhibit penetration of materials except for metals [1]. Many applications using THz light have been attempted in various fields such as semiconductors, biological materials, and polymers [2-6]. Among them, industrial applications are limited so far, and a few, such as characterization of paint films [7] and tablet coatings [8], were reported.

Rubber products, such as automobile tires, rubber bands, rubber balls, insulators, and O-rings, are essential to modern society. Manufacturers of these products use sophisticated chemical reagents and control systems; however, the manufacturing processes depend considerably on empirical procedures and craftsmanship. This situation suggests that the production is not very efficient and could be improved. Hence, to obtain better quality of rubber products, it is crucial to realize uniform dispersions of filler materials and to visualize vulcanization.

Our research group has been developing novel evaluation methods using $\mathrm{THz}$ radiation. $\mathrm{THz}$ absorbance spectra of various chemical materials used in rubber products has been reported $[9,10]$, and visualization of the dispersion of carbon black (CB), a major filler material, has been experimentally demonstrated [10]. It has also been reported that $\mathrm{THz}$ light could nondestructively detect the mesh network structure in the vulcanization reaction and the depth of the cure condition in rubber products having thicknesses greater than $10 \mathrm{~mm}$ [10]. These techniques are based on the transmission capability of $\mathrm{THz}$ radiation.

In this article, improved imaging of vulcanization depths in thick rubber samples and, for the first time, visualization of silica filler dispersion are demonstrated. The latter has been difficult to perform with conventional techniques. 


\section{Experimental}

THz time-domain spectroscopy (THz-TDS) was performed with a custom instrument (Otsuka Electronics Co., Ltd., TR-100KS) that included a femtosecond (fs) fiber laser (IMRA AMERICA, INC., Femtolite, $100 \mathrm{fs}, 1620 \mathrm{~nm}$ ) and a 4'-dimethylamino-N-methyl-4-stilbazolium tosylate (DAST) crystal for $\mathrm{THz}$ generation. Due to the $\mathrm{THz}$ detector of a photoconductive switch, the bandwidth of the applicable $\mathrm{THz}$ radiation was limited to $3 \mathrm{THz}$ for a better signal-noise ratio. The THz-TDS system was described in detail previously [10]; the transmission mode was used in this study.

The rubber samples were based on the synthetic polymer styrene-butadiene rubber (SBR, commercialgrade), which is widely used in automobile tires, and isoprene rubber (IR, commercial-grade). Table 1 lists the compounds in the samples used for the vulcanization imaging in thick rubber; all the reagents related to vulcanization except for filler materials used for high-sensitivity detection of mesh structure growth. The unit of "phr" in Table 1 is the preferred unit used in the rubber industry, indicating "parts per hundred rubber", which is the relative weight when the rubber polymer is 100. Samples for silica dispersion visualization had a different formulation, as shown in Table 2. The main difference from Table 1 was silica filler material, and a silane coupling reagent, which improves filler dispersion.

Table 1. Compounding formulation of the thick sample for vulcanization evaluation.

\begin{tabular}{ccc}
\hline Ingredient & Material & Quantity [phr] \\
\hline Polymer & SBR & 100 \\
Accelerator activators & Stearic Acid & 1 \\
& Zinc Oxide & 5 \\
Vulcanization accelerators & TMTD & 1 \\
& MBTS & 1 \\
Vulcanizing agent & CBS & 1 \\
\hline
\end{tabular}

Materials included styrene-butadiene rubber (SBR), tetramethlthiuram disulfide (TMTD), dibenzothiathiazole disulfide (MBTS), and n-cyclohexyl-2-benzothiazole sulfenamide (CBS).

Table 2. Compounding formulation of silica sample.

\begin{tabular}{ccc}
\hline Ingredient & Material & Quantity [phr] \\
\hline Polymer & IR or SBR & 100 \\
Filler & Silica & $30-130$ \\
Coupling agent & TESPT & Weight ratio of $8 \%$ of Silica \\
Accelerator activators & Stearic Acid & 2 \\
& Zinc Oxide & 2.5 \\
Vulcanization accelerators & DPG & 1.5 \\
& MBTS & 2.5 \\
Vulcanizing agent & TBBS & 0.7 \\
Others & Sulfur & 1.6 \\
& Wax & 2 \\
& Antioxidant & 1 \\
\hline
\end{tabular}

Materials included isoprene rubber (IR), styrene-butadiene rubber (SBR), diphenylguanidine (DPG), triethoxylpropyl tetrasulfide (TESPT), dibenzothiathiazole disulfide (MBTS), and N-(tert-butyl)-2-benzothiazole sulfenamide (TBBS).

The samples in Table 1; Table 2 were vulcanized via different methods. The sample for visualizing thick rubber curing was made with a mold, illustrated in Figure 1, that cured samples $35 \mathrm{~mm}$ in diameter and $20 \mathrm{~mm}$ thick. The vulcanization conditions were a pressure of $10 \mathrm{MPa}$ and a temperature of $150^{\circ} \mathrm{C}$. For THz-TDS analysis, the cured thick rubber sample was sliced into six $2-4$-mm-thick pieces, as shown in Figure 2. For THz absorbance spectra, 25 points in the central part of each sliced piece were evaluated. In the case of silica dispersion, masterbatch mixing, final mixing samples, and cured samples were used and imaged with a scanning electron microscope (SEM, CARL ZEISS AG, Ultra55), in addition to the $\mathrm{THz}$-TDS characterization. The cure conditions were $10 \mathrm{MPa}$ pressure at $160^{\circ} \mathrm{C}$. Here, we describe the 
experimental results of visualization of vulcanization and filler dispersion. These two conditions affect the quality of the rubber products considerably, and they are the main subject of concern of rubber manufactures; however, it was difficult to obtain their information rapidly and destructively using conventional techniques.

Filler dispersion was divided into two categories, micro-dispersion and macro-dispersion. Microdispersions were sub- $\mu \mathrm{m}$-sized aggregates that could be observed by an electron microscope. Here, the emphasis was on macro-dispersions of $\mathrm{mm}-\mu \mathrm{m}$-sized aggregates that could be evaluated visually or with an optical microscope. However, an established method has not been developed for objective numerical evaluations. As previously reported [10], the THz-TDS technique has the potential to observe the macro-dispersions of CB filler. In this study, the silica macro-dispersion was visualized.

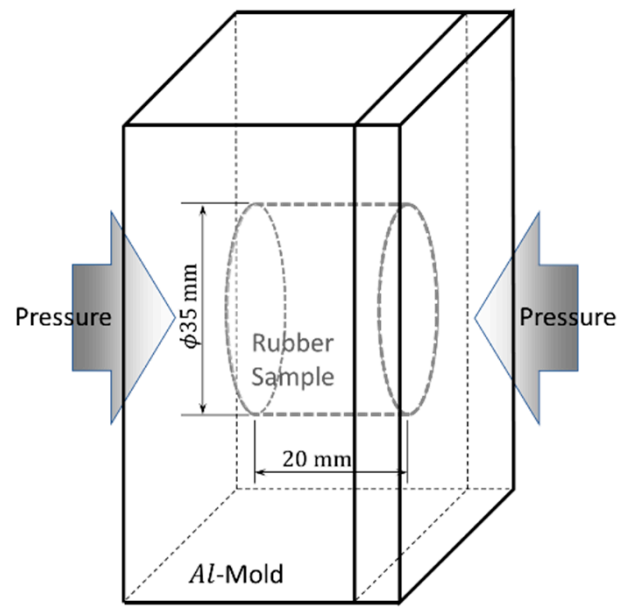

Figure 1. Mold for thick rubber production.

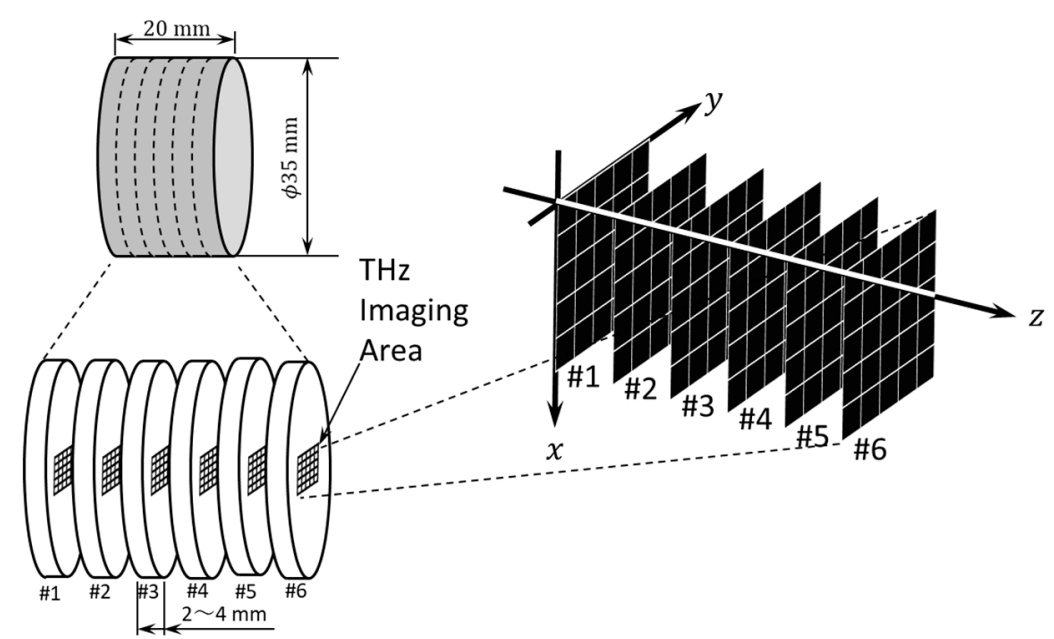

Figure 2. Sample preparation and measured areas for THz-TDS evaluation of thick rubber.

\section{Data Processing}

The THz-TDS data were evaluated as the absorbance $A$ per unit thickness $(\mathrm{cm})$ using

$$
A(v)=\frac{\log \frac{I_{0}(v)}{I(v)}}{T}\left[\mathrm{~cm}^{-1}\right]
$$

where $I_{0}$ and $I$ are the reference and transmitted THz light intensities through the sample, respectively, and $T$ is the sample thickness. After the $\mathrm{THz}$ absorbance spectrum was obtained at position $(x, y)$ on 
the sample, the representative value $A_{\text {rep }}(x, y)$ of the $\mathrm{THz}$ absorbance for the imaging process was calculated by

$$
A_{\text {rep }}(x, y)=\int_{v_{1}}^{v_{2}} \frac{A_{x y}(v) d v}{v_{2}-v_{1}}
$$

where $v_{1}$ and $v_{2}$ were the low- and high-end frequencies of the $\mathrm{THz}$ absorbance spectrum, respectively. The statistical values, such as mean, standard deviation (SD), and coefficient of variation (CV), were derived by $A_{\text {rep }}(x, y)$ s for one sample. $\mathrm{CV}$ defined by $\mathrm{SD} /$ mean was useful for measuring the degree of uniformity of the $A_{\text {rep }}(x, y)$ values over the sample. The penetration depth of the rubber samples used in this study was estimated to be about $1 \mu \mathrm{m}$. The transmission loss due to Fresnel reflections from rubber-air interfaces was estimated to $4 \sim 5 \%$ per interface; however, all the results processed in this study were calculated without considering this loss. This is because it was difficult to obtain an accurate loss for each rubber sample, and it was expected that the magnitude of the loss was not so critical when the $\mathrm{THz}$ transmission was evaluated as absorbance having a logarithm scale.

To estimate the dispersion of the silica filler, a new parameter evaluating fluctuations in the $\mathrm{THz}$ absorbance spectral profiles was introduced. Usually, the coefficient of correlation is used for this purpose; however, differences of the coefficient were small when applied to fluctuations of the absorbance spectra. Therefore, spectral profile dissimilarity (SPD) was used instead. Specifically, nine or 25 points in each sample were measured to evaluate the filler dispersion, and the SPD numerically calculated differences in the spectra by

$$
S P D=\frac{\sum_{\substack{j=1 \\(j \neq k)}}^{n}\left[\frac{\sum_{i=1}^{m}\left|\frac{A_{k}\left(v_{i}\right)-A_{j}\left(v_{i}\right)}{A_{k}\left(v_{i}\right)}\right|}{m} /\left|r_{k j}\right|\right]}{n-1}
$$

where $A_{k}(v)$ and $A_{j}(v)$ are the absorbances of the standard and compared spectra, respectively, as shown in Figure 3a. The $r_{k j}$ is Pearson's coefficient of correlation, which is usually $0.9 \mathrm{for} \mathrm{THz}$ absorbance spectra. Once the SPD value for one particular standard spectrum was obtained, the next standard spectrum was selected to calculate the next SPD. If $n$ points were sampled in one sample, the SPD s were averaged like,

$$
S P D_{\text {sample }}=\sum_{k=1}^{n} \frac{S P D_{k}}{n}
$$

Figure $3 \mathrm{~b}$ shows an example of $n=4$. Here, the $S P D$ values were indicated as 100 times $S P D$, $100 S P D$. For complete overlaps between all spectra, 100SPD takes the null value.

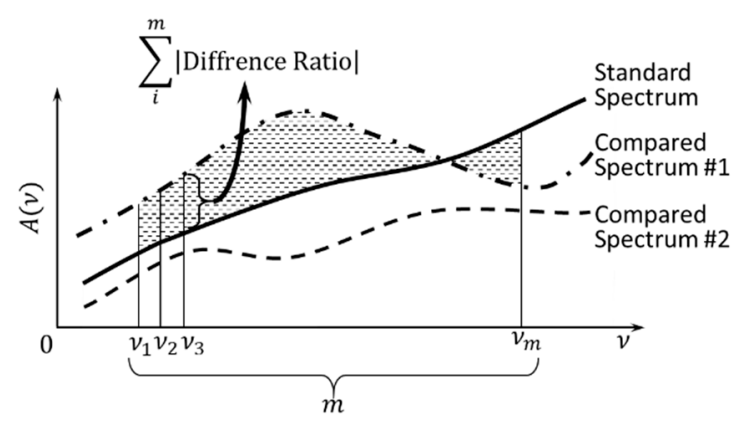

(a)

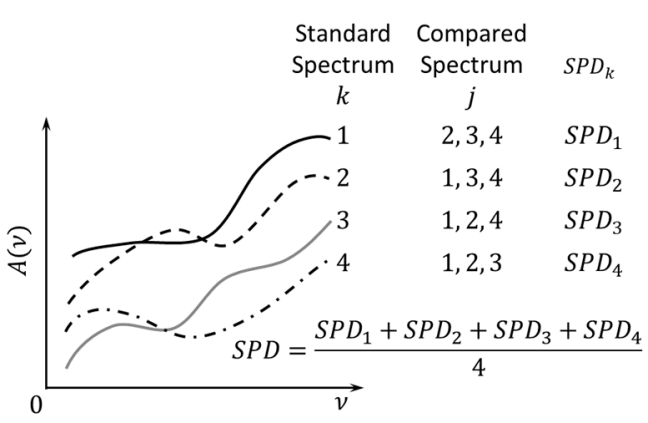

(b)

Figure 3. Definition of spectral profile dissimilarity (SPD). (a) SPD calculation, and (b) averaged SPD in one sample. 
To confirm the filler dispersion evaluation via $\mathrm{THz}$ absorbance, the silica dispersions were estimated with SEM. In the rubber industries, the macro-dispersion of filler in SEM images is usually evaluated with number or weight averaging of aggregates, which is correctly cross-section weight aggregate cross-section. This cross-section is calculated by using processed SEM images where the aggregate cross-section thresholds were $1 \mu \mathrm{m}^{2}$ and $10 \mu \mathrm{m}^{2}$, for example. The filler dispersion was evaluated using a statistical parameter for an accurate estimation. The discrepancy $D$ in the $k-$ dimensional unit cube is defined as $[11,12]$

$$
D_{N}^{(k)}=\sup _{t \in[0,1]^{k}}\left|\frac{\#([0, t) ; N)}{N}-t_{1} \times \cdots \times t_{k}\right|, t=\left(t_{1}, \cdots, t_{k}\right),
$$

where \# is the counting function, $N$ is the number of random nodes, and $t$ is the point set. This value varies from 0 to 1 , where smaller values indicate better dispersion. Because calculating this discrepancy is cumbersome even on a computer [12], a "simplified discrepancy" was used based on the concept of the discrepancy in this study. The idea of the new parameter is shown in Figure 4 . The evaluated sample was equally divided into 16 areas to count the particle (filler) after image binarization. The ratio of filler number to the total filler number $N$ in the sample was calculated, it was then subtracted by the normalized area section in each divided area, and its absolute value was calculated. Finally, the "simplified discrepancy" was obtained as the mean value over the 16 divided areas. The equation is as follows:

$$
D_{\text {simple }}=\frac{\sum_{i}^{n \mid}\left|\frac{\#\left(\text { area }_{i}\right)}{N}-S_{\text {norm }}\right|}{n},
$$

where $n$ and $S_{\text {norm }}$ are the divided area number in one SEM image and the normalized area cross-section, respectively. Here, the arithmetic mean was used instead of the operator "sup" in Equation (5), because the considered regions in one sample were only 16. This "simplified discrepancy" had values within a range of 0 to $(n-1) / n^{2}$, where 0 indicates complete uniformity.

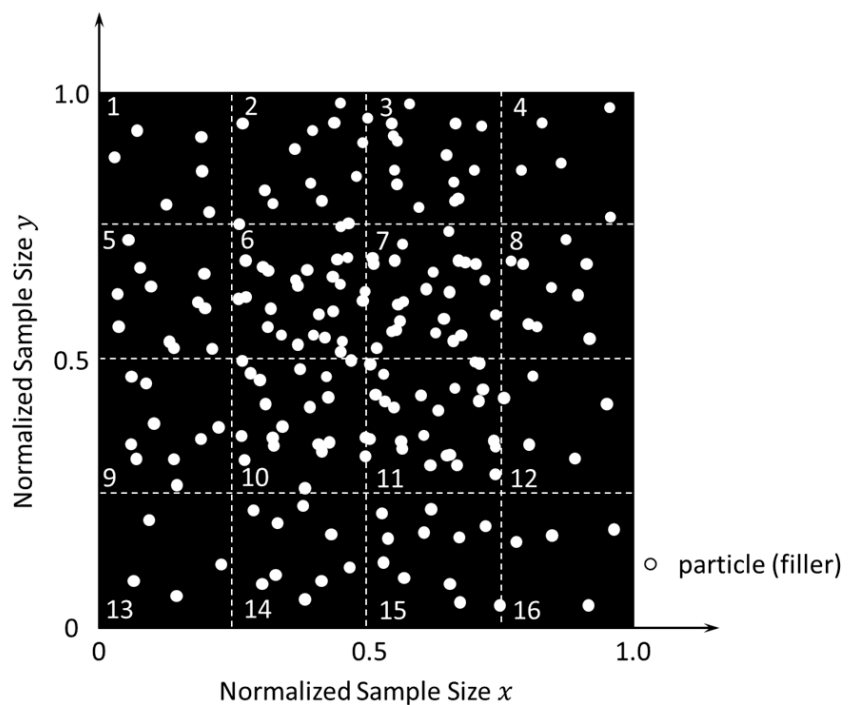

Figure 4. The concept of the simplified discrepancy calculation. This figure shows an example of $n=16$.

\section{Results and Discussion}

\subsection{Visualization of Vulcanization in Thick Samples}

A simple analysis of vulcanization conditions in thick samples has been reported previously [10]. Prior to the analysis of vulcanization depth by the $\mathrm{THz}$ light, the dependence of $\mathrm{THz}$ absorbance on the cure time was investigated. This procedure yielded two cure times, T90 and T100, for visualizing vulcanization determined by a curemeter (JSR Trading Co., Ltd. (JSR Corporation), CURELASTOMER 
W). T90 is generally called the "optimum vulcanization time," while T100 is the vulcanization finishing time. This simple analysis indicated that the central part of the sample had a low cure speed, while the $\mathrm{THz}$ absorbance in the center of the $\mathrm{T100}$ sample was higher than that for T90. The analysis only considered heat sources for vulcanization located in the pressed direction, and the analysis target was six sliced pieces; however, the actual thick sample was surrounded by the aluminum mold shown in Figure 1. This means that the sample was heated from all directions, and the sliced pieces each had a distribution of vulcanization. To solve this problem, the analyzed areas were set as shown in Figure 5. The previously analyzed sliced areas were depicted as \#1-\#6 in Figure 5a. The averaged $\mathrm{THz}$ absorbance values in each sliced piece had been evaluated previously [10]. Here, a total of 150 measured points was classified into the three sections "around the center", the "central part", and the surroundings of each ("edge"), as shown in Figure 5b,c. Figure 6 compares the THz absorbance and the $\mathrm{CV}$ values of the $\mathrm{THz}$ absorbance in each section. The comparison results for "around the center" and the "edge" are shown in Figure 6a,b, while Figure 6c,d indicates the results for the central and the edge parts. For the T90 sample, the THz absorbance differences between the edge and "around the center" in Figure $6 c$ are more significant than those in Figure $6 \mathrm{a}$. The same tendency was observed in the CV comparisons in Figure 6b,d. Previous results showed that the $\mathrm{THz}$ absorbance was proportional to the cure progress [10]. Therefore, considering the distance from the heat source, the results here suggest that the vulcanization reaction in the edge part of the $T 90$ sample was active and inhomogeneous, while in the "around the center"/central parts, the reaction progress was slower [10]. For the T100 sample, there was an almost equal $\mathrm{THz}$ absorbance for the edge and the "around the center"/central parts. This was understood by assuming that the cure reaction was nearly finished, and as a result, the CV values of the edge became small. Furthermore, the edge part in T100 samples indicated considerably larger CV values than that of the "around the center"/central parts. This result probably reflected that over-cure [10], which causes mesh structure destruction, occurred in T100 samples. Thus, it was confirmed that these detailed analyses revealed more information about vulcanization inside the thick rubber product.

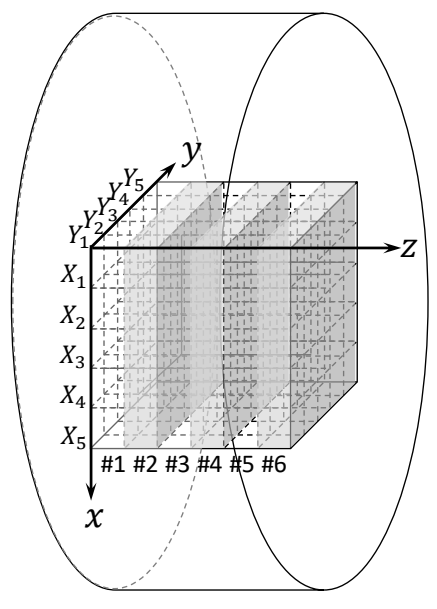

(a)

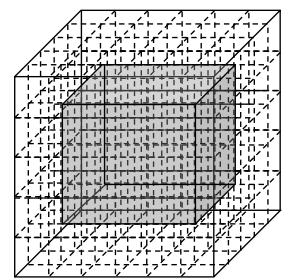

(b)

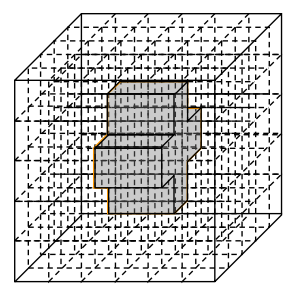

(c)

Figure 5. Thick rubber sample for detailed analysis in this study. (a) Coordinate axes and sliced areas (\#1 \#6) in the sample, (b) the "around the center" (36 points) and its surroundings ("edge," 114 points), and (c) the center (10 points) and its surroundings ("edge," 140 points). 


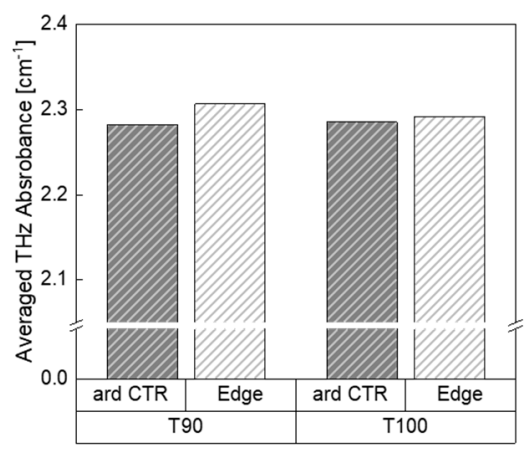

(a)

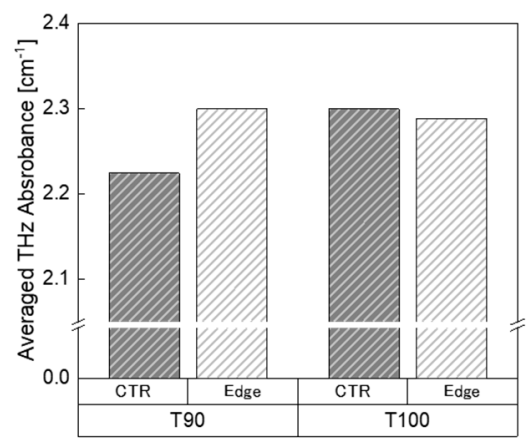

(c)

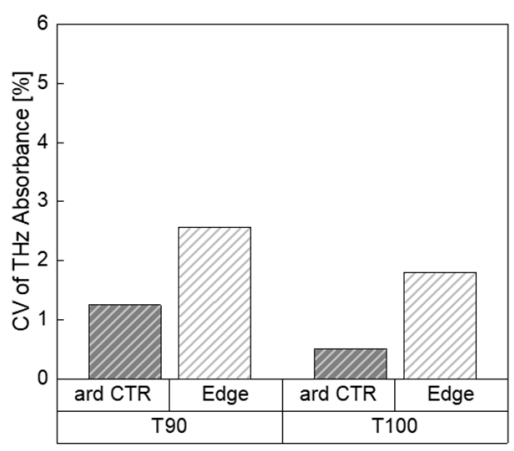

(b)

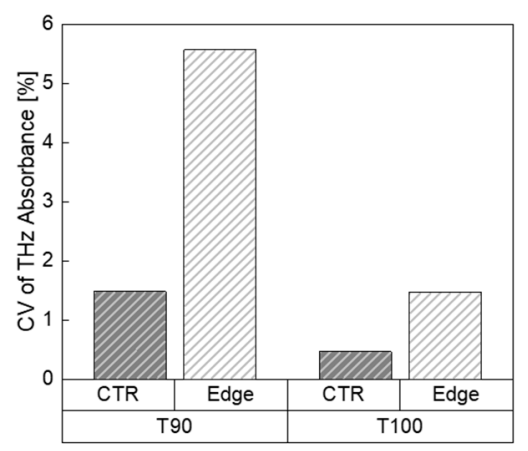

(d)

Figure 6. Comparison of the $\mathrm{THz}$ absorbance and the $\mathrm{CV}$ values of the $\mathrm{THz}$ absorbance in each section of T90 and T100 samples. (a) and (c) averaged THz absorbance in "around the center" and central part, and (b) and (d) CV of THz absorbance in "around the center" and central part, respectively.

\subsection{Silica Dispersion in Rubber Samples}

Previously the dispersion visualization of $\mathrm{CB}$, the most general filler in rubber, was reported [10]. Here, silica was visualized because it is a significant filler in ecological automobile tires. Because several unmeasured additives were used in the samples, $\mathrm{THz}$ absorbance spectra were evaluated for each of the new additives, as shown in Figure 7. The identification of observed peaks was not performed in this study. By considering the amount of each reagent used in Table 2, it was found that the diphenylguanidine (DPG), antioxidant, and wax were negligible in the $\mathrm{THz}$ absorbance of the rubber sample as much as other vulcanization accelerators and vulcanizing agent. Silane is an essential chemical material when silica is used as a filler in rubber products because silica cannot be uniformly distributed due to silanol groups on the silica surface. The silane bis(3-Triethoxylpropyl) tetrasulfide (TESPT in Table 2) reacted with silica at temperatures less than $140^{\circ} \mathrm{C}$, shown in Figure $8[13,14]$. The THz absorbance change due to this reaction was monitored in a preliminary experiment that used powdered samples. Because the bulk density of the powdered silica was approximately one-half that of the tablet silica, in addition to a scattering of $\mathrm{THz}$ light by powdered silica, the measured absorbance was smaller than that in Figure 7. When liquid silane was mixed with the silica, the silica tended to clump, and the absorbance spectrum was obtained by averaging three points. In contrast, the absorbance of the pure silica powder sample was stable. After heating at $130^{\circ} \mathrm{C}$ for $2 \mathrm{~h}$, the spectra of the mixed powder were as stable as those of the pure silica; however, the $\mathrm{THz}$ data were measured at the different three points in the sample and averaged. From Figure 9, it was found that the absorbance increased with silane addition and, after heating (silica-silane reaction), the absorbance decreased. Although the cause of this decrease was unclear (it may be an intermediate product, shown in Figure 8), the results indicated the detection of the silica inside rubber samples. 


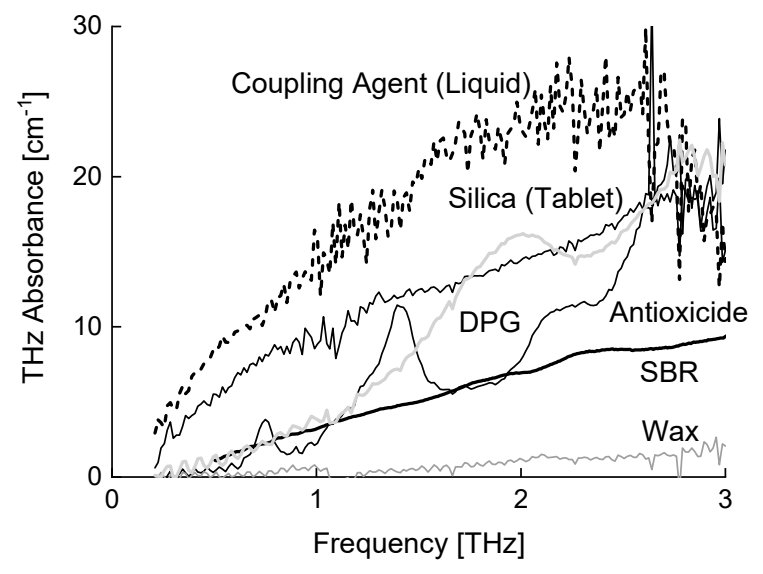

Figure 7. THz absorbance spectra for newly measured agents.

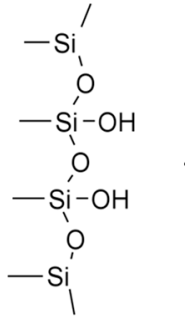

Silica

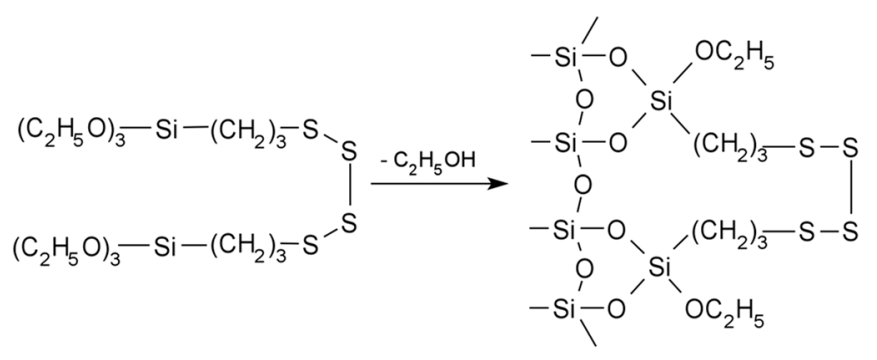

Silane (TESPT)

\section{Silica/TESPT intermediate product}

Figure 8. Chemical reaction between silica and silane.

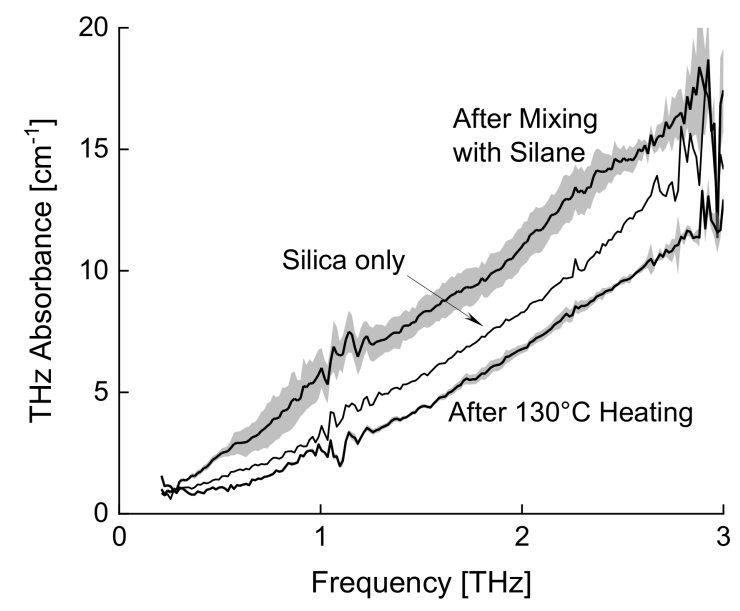

Figure 9. Change of the $\mathrm{THz}$ absorbance spectrum by silica-silane chemical reaction. Gray-colored zones mean standard errors.

A calibration curve of the silica $\mathrm{THz}$ absorbance was used to visualize the dispersion of silica filler inside the rubber samples. Samples made from two different polymers were prepared because SBR polymer, which is the main target and widely used in automobile tires, has a lower viscosity and affects filler dispersion. Isoprene rubber (IR) was selected for comparison because it has a simple structure, a higher Mooney viscosity (stiffer), and an almost equal THz absorbance relative to SBR. When the calibration curves were calculated for silica filler, the same procedure was chosen as that for CB filler dispersion [10]. The THz-TDS system evaluated 25 points in each sample and processed the data to produce absorbance images. The results are shown in Figure 10. Both the IR and SBR samples had excellent correlations between the THz absorbances and the amount of silica. The squared Pearson's 
coefficient of correlation, $r^{2}$, was 0.994 and 0.990 for IR and SBR, respectively. Comparing the previous results using $C B$ filler, no saturation was observable at higher silica amounts. This suggests that internal scattering or multiple scattering inside the rubber samples was not dominant even in samples having higher filler concentration. This assumption was supported by the fact that remarkable temporal $\mathrm{THz}$ waveform degradation [15] was not observed in samples containing silica filler (the waveform is not shown). The parameters of $C V$ and SPD were plotted together in Figure 10. The $C V$ was obtained from the integrated absorbance values, which eliminated the $\mathrm{THz}$ absorbance spectral fluctuations. In contrast, the $S P D$ reflected the difference in spectral profiles. Increased filler concentration induced more uniform dispersion; thus, the $C V$ and SPD trends in Figure 10 were acceptable. Furthermore, the variations were comparable. Both samples indicated minimum values at $70 \mathrm{phr}$ silica; however, the cause was not understood. The 70-phr silica possibly matched the quantities of other additives and polymer weights. Figure 10 verified that $\mathrm{THz}$ absorbance was suitable for evaluating the silica dispersion, and that the parameter $S P D$ was a potential indicator of spectral uniformity.

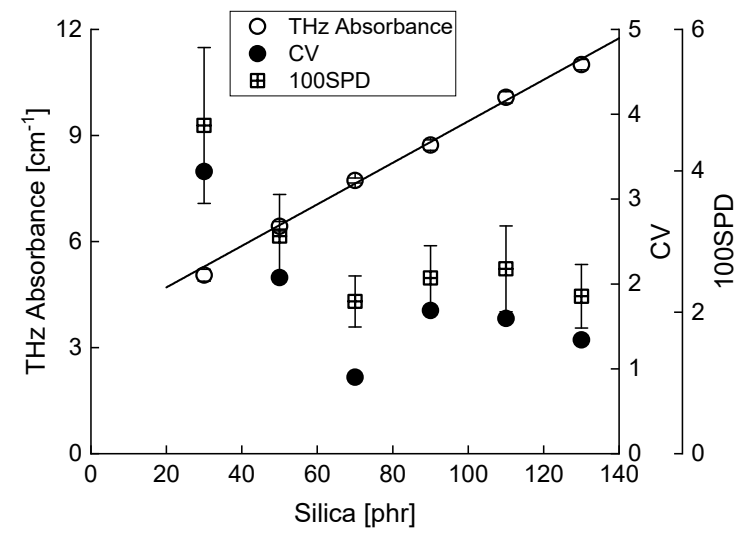

(a)

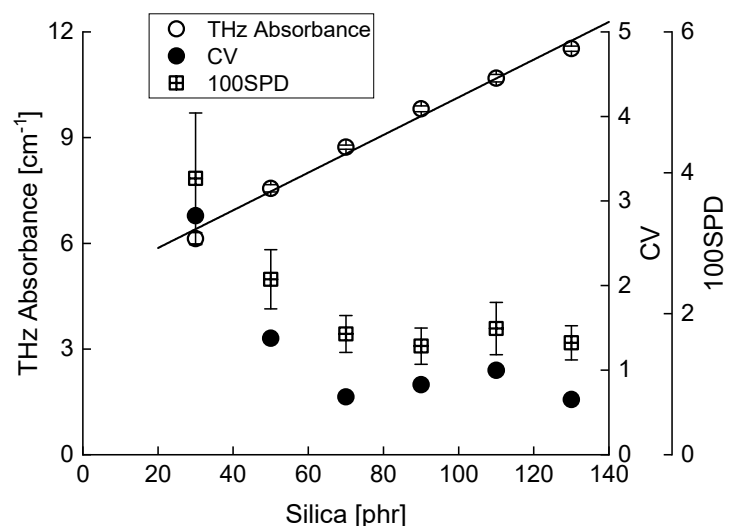

(b)

Figure 10. Calibration lines for the $\mathrm{THz}$ absorbance (solid line) against silica concentration with $C V$ of the THz absorbance and 100SPD of the THz absorbance spectra. (a) IR- and (b) SBR-based sample.

The visualization of silica dispersion was performed using the same imaging procedure as that used for CB filler [10]. The grayscale images for the silica dispersion were inferior to the CB dispersion images in recognition of the dispersion. Meanwhile, the fluctuation of the absorbance spectrum was suitable to evaluate silica dispersion in the samples. This was because there was much less silica or silica-silane compound absorption relative to that of $\mathrm{CB}$, although the linearity of the silica absorbance was confirmed in Figure 10. The 25 spectra were overlapped in one plot in Figure 11 to identify fluctuations more easily. The $\mathrm{THz}$ absorbance image is shown in the inset of each spectrum for reference. The spectra were for (a) silica masterbatch mixing (unvulcanized), (b) final mixing (unvulcanized), and (c) vulcanized rubber. These production processes of rubber products, such as masterbatch mixing and final mixing, were explained previously [10]. The first process was the masterbatch mixing, and the last condition of the product was a vulcanized rubber, which follows the final mixing. As shown in Figure 11, the fluctuations settled as the procedure progressed. The SPD in Equations (3) and (4) was used to evaluate the spectral fluctuations. Table 3 summarizes the averaged absorbance, $C V$, and 100SPD for these three conditions over the range 1.2-2.6 THz. The mean value of the absorbance was almost constant, while the fluctuations gradually decreased with the process. To confirm the validity of the fluctuation evaluation with the SPD parameter, SEM images were acquired for samples of masterbatch mixing and cured rubber. The SEM images and the analysis of dispersion by the simplified discrepancy are shown in Figure 12. The brightness of the images was slightly adjusted by software to emphasize the surface conditions for the (a) masterbatch mixing and (b) cured rubber. In Figure 12b, the vulcanized rubber had a clean surface. The evaluation by the simplified discrepancy is shown in Figure 12c, which has two bars for the masterbatch mixing sample (from two SEM images) and one bar for the cured rubber. The data confirmed that the value of the 
simplified discrepancy for the cured sample was smaller with fewer errors than that of the masterbatch sample, and that the dispersion evaluation of silica filler by $\mathrm{THz}$ absorbance was valid. This result proved that the novel parameter of the simplified discrepancy can function for the SEM image analysis.

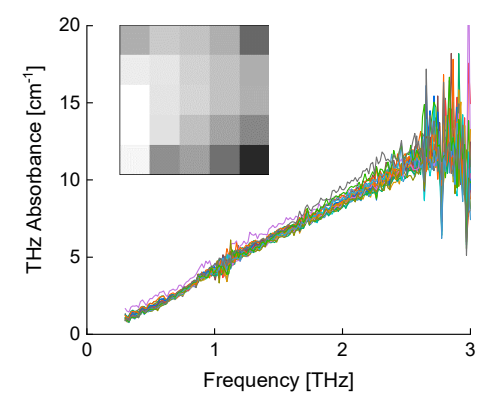

(a)

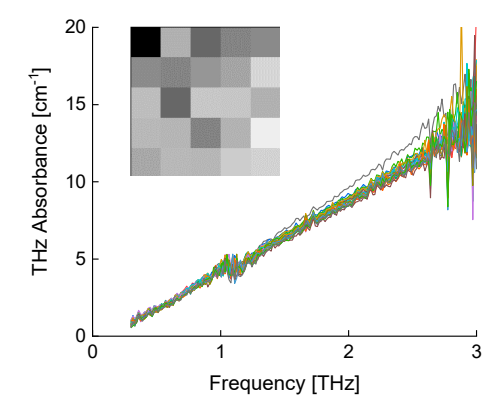

(b)

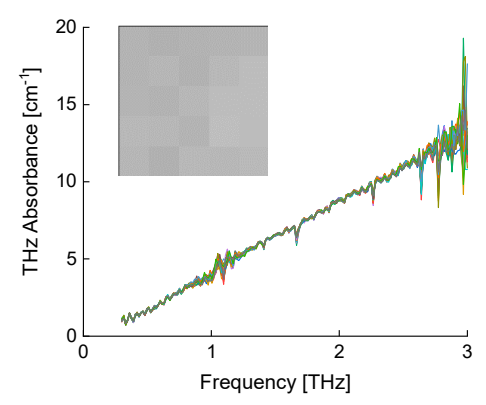

(c)

Figure 11. THz absorbance spectra at 25 measured point in various sample condition. (a) Silica masterbatch mixing (unvulcanized), (b) final mixing (unvulcanized), and (c) vulcanized rubber. Inset shows each $\mathrm{THz}$ absorbance image.

Table 3. The averaged $\mathrm{THz}$ absorbance, $\mathrm{CV}$, and 100SPD for masterbatch mixing, final mixing, and vulcanized rubber.

\begin{tabular}{cccc}
\hline Sample & Masterbatch Mixing & Final Mixing & Cured Rubber \\
\hline Mean & 8.20 & 8.32 & 8.22 \\
CV [\%] & 3.04 & 2.84 & 0.242 \\
$100 S P D$ & 6.32 & 5.68 & 2.38 \\
\hline
\end{tabular}

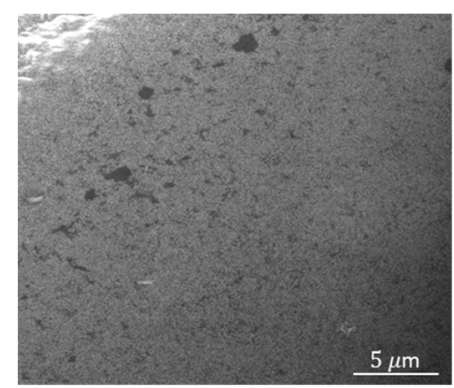

(a)

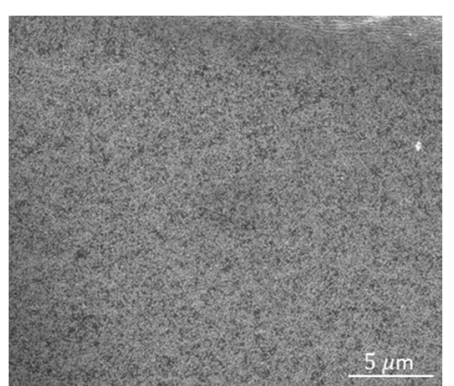

(b)

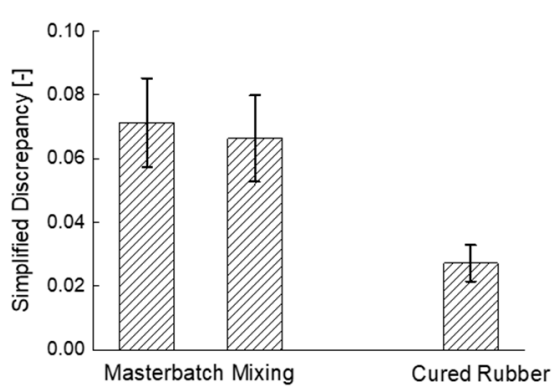

(c)

Figure 12. SEM image evaluation of SBR-based sample. (a) Silica masterbatch mixing, (b) vulcanized rubber, and (c) silica dispersion in SEM images derived by simplified discrepancy.

\section{Conclusions}

The vulcanization condition inside thick rubber samples was visualized by $\mathrm{THz}$ absorbance images in detail. They revealed that the surrounding aluminum mold acted as a heat source, and that the central and the "around the center" regions had a lower rate of vulcanization relative to the edges. The $\mathrm{THz}$ absorbance characterized the dispersion of the silica filler in the rubber samples, which is difficult to evaluate nondestructively and rapidly with other methods. The profile fluctuation of the absorbance spectrum was an effective indicator of the homogeneity of the silica filler. The validity of this method was confirmed with SEM imaging, where a new parameter was used to evaluate the filler uniformity. Overall, the $\mathrm{THz}$ technique could be used for detecting or assessing the distribution of silica, which will have more importance in future rubber products. 
Author Contributions: Conceptualization, M.H., T.G and Y.H.; methodology, T.G. and Y.H.; software, Y.H.; validation, M.H., T.G. and Y.H.; formal analysis, Y.Y., T.M. (Toshiaki Morichika), R.S., T.M. (Takakazu Minato) and Y.H.; investigation, Y.Y., R.S., T.M. (Toshiaki Morichika), T.M. (Takakazu Minato) and Y.H.; resources, M.H., T.G and Y.H.; data curation, M.H., T.G., Y.Y. and Y.H.; writing-original draft preparation, Y.H.; writing-review and editing, Y.Y., T.G., R.S., T.M. (Toshiaki Morichika), T.M. (Takakazu Minato), M.H. and Y.H.; visualization, Y.H.; supervision, M.H. and Y.H.; project administration, M.H. and Y.H.; funding acquisition, M.H. All authors have read and agreed to the published version of the manuscript.

Funding: This research received no external funding.

Acknowledgments: The authors thank associate professor Katsunori Watanabe and technical staff member Takuta Kamino, the national institute of technology (KOSEN), Kurume College, for helping in making the rubber compounds. We thank Alan Burns, from the Edanz Group (www.edanzediting.com/ac) for editing a draft of this manuscript.

Conflicts of Interest: The authors declare no conflict of interest.

\section{References}

1. Tonouchi, M. Cutting-edge terahertz technology. Nat. Photonics 2007, 1, 97-105. [CrossRef]

2. Schmuttenmaer, C.A. Exploring Dynamics in the Far-Infrared with Terahertz Spectroscopy. Chem. Rev. 2004, 104, 1759-1780. [CrossRef] [PubMed]

3. Heilweil, E.J.; Plusquellic, D.F. Terahertz Spectroscopy of Biomolecules. In Terahertz Spectroscopy Principles and Applications; Dexheimer, S.L., Ed.; CRC Press: Boca Raton, FL, USA, 2007; pp. 269-298.

4. Hoshina, H.; Hayashi, A.; Miyoshi, N.; Miyamaru, F.; Otani, C. Terahertz pulsed imaging of frozen biological tissues. Appl. Phys. Lett. 2009, 94, 123901. [CrossRef]

5. Rungsawang, R.; Geethamma, V.G.; Parrot, E.P.J.; Ritchie, D.A.; Terentjev, E.M. Terahertz spectroscopy of carbon nanotubes embedded in a deformable rubber. J. Appl. Phys. 2008, 103, 123503. [CrossRef]

6. Piesiewicz, R.; Jansen, C.; Wietzke, S.; Mittleman, D.; Koch, M.; Kürner, T. Properties of Building and Plastic Materials in the THz Range. Int. J. Infrared Millim. Waves 2007, 28, 363-371. [CrossRef]

7. Yasui, T.; Yasuda, T.; Sawanaka, K.; Araki, T. Terahertz paintmeter for noncontact monitoring of thickness and drying progress in paint film. Appl. Opt. 2005, 44, 6849-6856. [CrossRef] [PubMed]

8. Fitzgerald, A.J.; Cole, B.E.; Taday, P.F. Nondestructive analysis of tablet coating thicknesses using terahertz pulsed imaging. J. Pharm. Sci. 2005, 94, 177-183. [CrossRef] [PubMed]

9. Hirakawa, Y.; Ohno, Y.; Gondoh, T.; Mori, T.; Takeya, K.; Tonouchi, M.; Ohtake, H.; Hirosumi, T. Nondestructive Evaluation of Rubber Compounds by Terahertz Time-Domain Spectroscopy. J. Infrared Millim. Terahertz Waves 2011, 32, 1457-1463. [CrossRef]

10. Hirakawa, Y.; Yasumoto, Y.; Gondo, T. Evaluation of Rubber Products by Terahertz Time-domain Spectroscopy Carbon Black Dispersion and Vulcanization State. J. Infrared Millim. Terahertz Waves 2020. [CrossRef]

11. Niederreiter, H. Quasi-Monte Carlo Methods for Numerical Integration. In Random Number Generation and Quasi-Monte Carlo Methods; Society for Industrial and Applied Mathematics: Philadelphia, PA, USA, 1992; pp. 13-22.

12. Gandar, B.; Loosli, G.; Deffuant, G. Sample Dispersion Is Better than Sample Discrepancy for Classification, hal-00679061. 2010. Available online: https://hal.archives-ouvertes.fr/hal-00679061 (accessed on 1 February 2020).

13. Rodgers, B.; Wanddell, W. The Science of Rubber Compounding. In The Scientific and Technology of Rubber, 4th ed.; Mark, J.E., Erman, B., Roland, C.M., Eds.; Academic Press: Amsterdam, The Netherlands, 2013; pp. 417-471.

14. Ichino, T. Silane Coupling Agent for Rubber Usage. J. Soc. Rubber Sci. Technol. Jpn. 2009, 82, 67-72. [CrossRef]

15. Hirakawa, Y.; Yamauchi, T.; Kamino, T.; Gondoh, T.; Hirano, S.; Noguchi, T. Dependence of THz Signals on Carbon Black Compounding Amount in Vulcanized Rubber. In Technical Digests of the Conference on Lasers and Electro-Optics (CLEO); Optical Society of America: San Jose, CA, USA, 2017. 